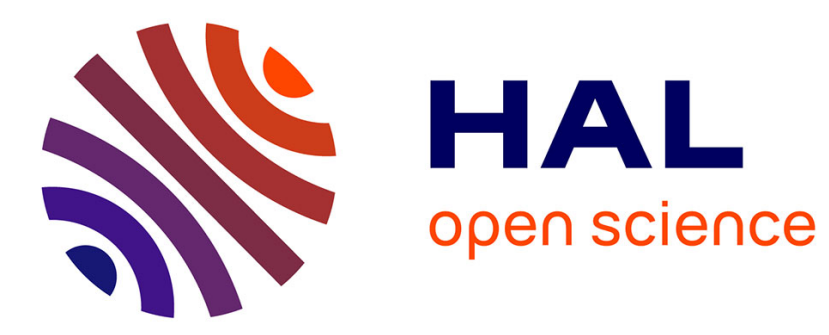

\title{
Sacrifices de la foule, Sacrifice de Judas: l'Évangile de Judas et le thème sacrificiel
}

\author{
Anna van den Kerchove
}

\section{To cite this version:}

Anna van den Kerchove. Sacrifices de la foule, Sacrifice de Judas: l'Évangile de Judas et le thème sacrificiel. Apocrypha, 2009, 20, pp.213-228. hal-00735836

\section{HAL Id: hal-00735836 \\ https://hal.science/hal-00735836}

Submitted on 26 Sep 2012

HAL is a multi-disciplinary open access archive for the deposit and dissemination of scientific research documents, whether they are published or not. The documents may come from teaching and research institutions in France or abroad, or from public or private research centers.
L'archive ouverte pluridisciplinaire HAL, est destinée au dépôt et à la diffusion de documents scientifiques de niveau recherche, publiés ou non, émanant des établissements d'enseignement et de recherche français ou étrangers, des laboratoires publics ou privés. 
Anna Van den Kerchove

Ecole pratique des hautes études/Institut européen en sciences des religions

\section{Sacrifices de la foule, Sacrifice de Judas : \\ l'Évangile de Judas et le thème sacrificiel ${ }^{\mathrm{I}}$}

Résumé / abstract :

Le thème sacrificiel, malgré le peu de pages qui lui est consacré, est important dans l'économie de l'Évangile de Judas. L'auteur y a recours une première fois au sein d'une liste d'actions accomplie par une foule qui représenterait ses adversaires. L'auteur détournerait l'usage qui est fait du langage sacrificiel dans d'autres communautés pour critiquer les rites et pratiques de ses adversaires, notamment l'eucharistie, la continence et peut-être le martyre. Par delà, il y aurait également une critique ecclésiologique. L'auteur utilise une seconde fois le langage sacrificiel pour parler de la trahison de Judas, et la question se pose de la valeur que l'auteur accorde à cet acte, ainsi que finalement à la figure de Judas.

Despite the few pages only which are dedicated to the sacrificial theme, it is important in the economy of the Gospel of Judas. The first time the author mentions sacrifices is within a list of actions accomplished by a crowd which may represent his opponents. The author probably diverts the use of the sacrificial language which is made in the other communities so as to criticize the rites and practices of his opponents, in particular eucharist, continence and maybe martyrdom. There may also be in the background an ecclesiological criticism. The author uses sacrificial language for a second time in order to speak about the treason of Judas, and the question arises of the value granted to this act and to the figure of Judas.

L'Évangile de Judas appartient aux nombreux courants exégétiques des événements et faits liés à Jésus, en particulier des personnages qui gravitent autour de lui, ici de Judas. Cette figure ne fait pas l'objet de

${ }^{\text {I }}$ Cet article est fondé sur la version française de l'article publié en lithuanien : "Minios aukos ir Judo auka: aukojimo tema Judo evangelijoje », ALEKNIENÉ T. et AKekna D., Saeculo primo: Romos imperijos pasaulis peržengus „naujosios eros “ slenksti (Christiana Tempora III), Vilnius, 2008, p. 274-294. Il complète notre autre article : «La maison, l'autel et les sacrifices : quelques remarques sur la polémique dans l'Évangile de Judas», Scopello M. (éd.),

The Gospel of Judas in Context. Proceedings of the First International Conference on the Gospel of Judas. Paris, Sorbonne, October $27^{\text {th }}-28^{\text {th }} 2006$ (Nag Hammadi and Manichaean Studies 62), Leyde, 2008, p. 3I I-330. 
longs développements dans les premiers textes chrétiens; cela n'empêche pas une évolution dans la conception que les auteurs se font de sa trahison, et l'Évangile selon Jean est, parmi les évangiles qui deviennent canoniques, celui qui discrédite le plus Judas². Il n'est pas dans notre intention de parler de Judas, en relation avec cette tradition $^{3}$, mais d'évoquer un thème afférent, celui des sacrifices. Ce thème est plus important qu'il n'y paraît à première vue. En effet, si l'auteur n'y consacre que quatre pages sur les vingt-cinq que compte son texte, il en parle à deux moments clé : une première fois aux pages 38-4I, quand les disciples exposent à Jésus leur vision de la maison et des activités qui s'y déroulent; une seconde fois à la fin du texte, page 56 , quand Jésus révèle à Judas ce que celui-ci devra faire dans un avenir proche : «mais quant à toi, tu feras plus qu'eux tous ; en effet, l'homme qui me porte, tu le sacrifieras », référence explicite à l'événement présenté comme une trahison de Judas par les évangiles qui deviennent canoniques et la tradition chrétienne qui en dépend. Ces deux passages soulèvent de nombreuses questions auxquelles nous ne pourrons pas répondre pour le moment; nous ne ferons qu'ébaucher des pistes de réflexion, en examinant, comment, à partir de ce thème, des événements et des faits du Ir siècle sont réinterprétés au $\mathrm{II}^{\mathrm{e}}$ siècle en relation avec le contexte spécifique de ce siècle et les intentions de l'auteur de cet Évangile de Judas.

Afin de bien comprendre ces intentions, rappelons rapidement le contexte de chacun des deux passages, pages 38-4I et page 56 . Dans le premier, les disciples prennent l'initiative de l'évocation des sacrifices, lorsqu'ils exposent leur vision à Jésus, et celui-ci ne fait que reprendre ce thème quand il interprète leur vision. Dans le second passage, c'est désormais Jésus qui a l'initiative de parler des sacrifices. Il ne s'agit plus d'une vision et de son exégèse, mais d'une part, d'un rappel fait par Jésus concernant un groupe d'hommes sacrifiant et d'autre part, de l'annonce à Judas de ses actions futures. Cette dernière mention a ainsi plutôt un caractère prophétique, alors que dans le premier passage, il s'agit d'un contexte de vision. Ceci a des conséquences sur la manière dont il faut lire et interpréter chacun de ces passages.

La vision des disciples : un cadre polémique

Dans le premier passage, en ÉvJudas 38. I-I I, les disciples débutent la description de leur vision ainsi :

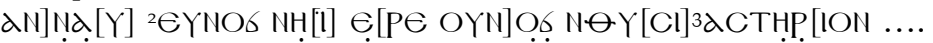
dy]

2 Robinson J. M., The Secrets of Judas. The Story of the Misunderstood Disciple and his lost Gospel, New York, Harper San Francisco, 2006, p. I-3I. ${ }^{3}$ Sur la figure de Judas dans l'Évangile de Judas, voir différents articles dans Scopello M. (éd.), The Gospel of Judas in Context, op. cit. 


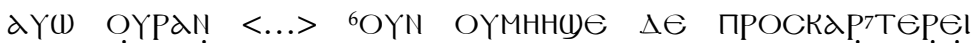

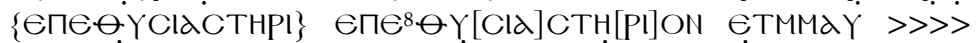

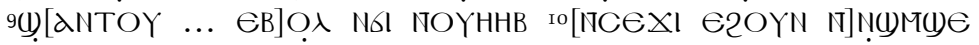

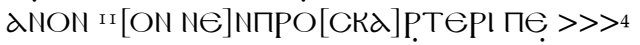

nous avons vu une grande maison [........] il y avait un grand autel, et douze hommes - nous disons que ce sont des prêtres - et un Nom ; et une foule s'active avec persévérance près de cet autel, jusqu'à ce que les prêtres .... et [présentent] des offrandes; nous [aussi], nous nous activons avec persévérance.

Les disciples débutent la description de leur vision en donnant le cadre général du contenu de cette vision. Dans un premier temps, lignes I à 5, ils énumèrent les éléments architecturaux et les principaux protagonistes: une maison, un autel, douze hommes qui, selon l'opinion des disciples, sont des prêtres, et un nom (il s'agit peut-être du nom de Dieu ou de Jésus; pour le moment, la réponse reste en suspens, même si nous pensons que l'auteur est volontairement ambigu sur l'identité de ce nom). Dans un second temps, lignes 6 à I I, les disciples évoquent les activités accomplies en relation avec la maison et l'autel et dont les disciples sont les spectateurs ; ils mettent en avant les prêtres, puis la foule, qui se tient devant l'autel, et enfin les disciples, qui se voient eux-mêmes. Pour décrire leur propre activité, les disciples utilisent le même verbe que pour la foule, ПРОСКגPTЄP€l « s'activer avec persévérance ${ }^{5} »$; se voient-ils au milieu de la foule ? En tout cas, ils se mettent en avant par rapport à la foule et ils se distinguent des prêtres. Tout le cadre général est centré autour du thème sacrificiel, avec les deux occurrences du terme $\theta$ YCldCTHPION${ }^{6}$ et la mention des offrandes (ÉvJudas 38.Io): l'espace et les activités qui s'y déroulent sont organisés autour de l'autel $\Theta$ YCldCTHPION ; la maison, $\mathrm{HI}$, est ainsi définie comme un lieu de culte, les douze hommes comme des prêtres (identification que les disciples font déjà), et l'activité de la foule prend une dimension nouvelle, rituelle, puisqu'elle est accomplie

${ }^{4}$ Nous adoptons en grande partie le texte de l'editio princeps: The Gospel of Judas together with the Letter of Peter to Philip, James, and a Book of Allogenes from Codex Tchacos - Critical Edition, Coptic Text edited by Rodolphe KASSER and Gregor WURST, Introductions, Translations, and Notes by Rodolphe Kasser, Marvin Meyer, Gregor Wurst and François GAUDARD, Washington D.C., 2007, sans reprendre toutefois toutes les restitutions proposées.

5 Par cette traduction, nous avons voulu rendre à la fois l'idée d'intensité et

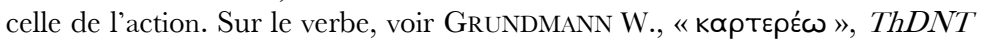
III, I974, p. 6I7-620.

${ }^{6} \mathrm{Si}$ on considère que l'occurrence du terme $€ \ominus$ $\ominus$ CldCTHPI page 38 , ligne 7 est bien une dittographie, comme le supposent Kasser R., Meyer M. and Wurst Gr., The Gospel of Judas, Washington D. C., National Geographic, 2006.p. 26 n. 38 . 
devant l'autel. La présence du Nom ne fait que renforcer cet aspect cultuel.

L'exposition du cadre général est suivie par une question de Jésus qui demande des précisions. Question lacunaire, nous proposons de la restituer de la manière suivante: $21 T M d(1) \operatorname{MMI}^{13}[\mathrm{~N} \in$ пе пMHH()E], «de quelle sort[e est la foule] ? » 7 . Les disciples énumèrent en une longue réponse ( $E_{V} J u d a s 38.13-39.3$ ) les actions que la foule (s'il s'agit bien d'elle) perpètre et qui explicitent la proposition des lignes 6-8 «s'activer avec persévérance près de cet autel» (ÉVJudas 38.1339.3):

nToOY $\Delta \in{ }^{2} 4\left[\Pi \in X d Y \quad X \in\right.$ 2OEl]ne MEN $\in Y^{15}[\ldots . .$.

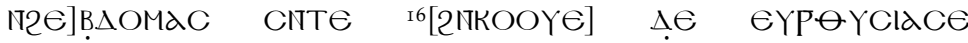

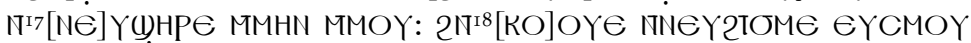

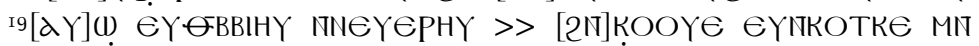

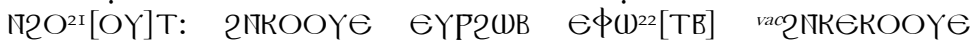

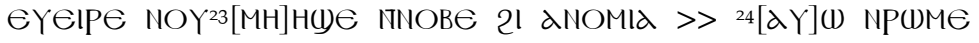

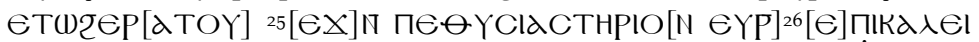

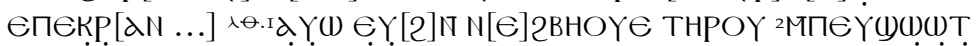

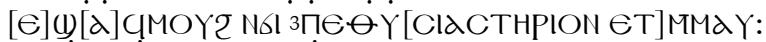

«Et eux, [ils dirent: "les u]ns [.....] deux semaines, tandis que les autres, ce sont leu[rs propres] enfants qu'ils sacrifient, d'[au]tres leurs femmes, tout en louant [e]t en étant humbles les uns envers les autres ; [d']autres dorment avec des ho[mm]es; d'autres commettent des meu[rtres], d'autres font un [gra]nd nombre de péchés et d'actions illégales ; [e]t les hommes qui se tiennent d[ebout dev] ant l'autel, c'est ton $\mathrm{n}[\mathrm{om}]$ qu'ils invoquent; et c'est alors qu'ils sont parmi toutes les actions de leur déficience que cet [autel] est complété”. »

Parmi toutes ces actions, nous pouvons en distinguer six principales : la première, malheureusement dans une lacune, pourrait être le jeûne ${ }^{8}$; les deux suivantes sont des sacrifices, d'enfants et de femmes, accomplis, au moins pour ceux des femmes, alors que les sacrifiants sont en train de louer et d'être humbles les uns envers les autres; la quatrième action est le fait de dormir avec d'autres hommes; la cinquième, les meurtres; et la dernière, les péchés et autres actions viles. Au sein de cette liste, les sacrifices tiennent une place qui n'est pas négligeable, puisqu'ils apparaissent deux fois sur six, une fois explicitement (sacrifices des enfants) et une seconde fois implicitement (sacrifice des femmes). L'auteur n'a pas établi cette liste au hasard, mais il l'a organisée selon une logique dont il faut donner la clef. Les

\footnotetext{
7 Pour cette restitution: VAN DEN Kerchove A., «La maison, l'autel et les sacrifices ", op. cit., p. 3I9.

8 Pour cette suggestion: VAN DEN Kerchove A., «La maison, l'autel et les sacrifices ", op. cit., p. 320. Elle a également faite dans l'editio princeps: The Gospel of Judas, op. cit., p. I95.
} 
actions énumérées répondent à deux critères : le caractère rituel ou non des actions et leur qualité, négative ou, a priori, positive. La combinaison de ces deux critères donne la répartition suivante :

\begin{tabular}{|l|l|l|}
\hline & Actions rituelles des & Actions non rituelles \\
\hline Actions négatives & $\begin{array}{l}\text { sacrifices sormir avec d'autres } \\
\text { enfants ; sacrifices } \\
\text { des femmes }\end{array}$ & $\begin{array}{l}\text { hommes meurtres ; } \\
\text { péchés et autres } \\
\text { actions viles }\end{array}$ \\
\hline Actions positives & $\begin{array}{l}\text { jeûner ( ?); louer; } \\
\text { être humble }\end{array}$ & $\begin{array}{l}\text { il n'y a pas d'action } \\
\text { non rituelle positive }\end{array}$ \\
\hline
\end{tabular}

L'auteur, par l'intermédiaire des disciples, commence la liste avec les actions rituelles avant d'énumérer celles qui, a priori, ne le sont pas et qui concernent essentiellement les mœurs. Cette succession d'actions toutes mises sur le même plan semble être un moyen d'assimiler les actions rituelles aux actions non rituelles et de dénier aux premières leur caractère rituel. De plus, les actions positives sont peu nombreuses par rapport aux actions négatives : une seule parmi les six actions principales, à savoir le jeûne - s'il s'agit bien de cela - et les deux actions secondaires, la louange et le fait d'être humbles les uns envers les autres. Ce faible nombre et l'association des deux actions secondaires avec une action négative, sacrifice de femmes, donnent une connotation largement négative à l'ensemble de la liste et aussi aux rares actions positives. Toute cette organisation ne peut donc se comprendre que si on considère qu'elle est élaborée dans une visée polémique : avec elle, l'auteur critiquerait en fait ses adversaires, des chrétiens qui penseraient accomplir des actes rituels alors qu'en réalité, selon lui, ces actes n'ont rien de rituel. Ce but polémique ne se retrouverait-il pas dans la présentation du cadre général, les activités de la foule étant accomplies en présence de l'autel de la maison?

Dans la présentation du cadre général, les disciples parlent d'une maison et d'un autel, mais la relation architecturale entre les deux reste dans l'ombre, une lacune intervenant malencontreusement à cet

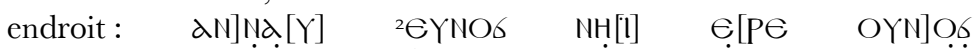

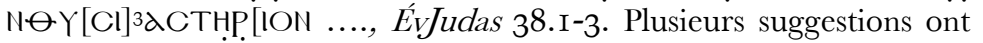
été faites, aussi valables les unes que les autres9. Déterminer le référent exact de la maison vue par les disciples pourrait orienter la solution. Cependant, là aussi, plusieurs possibilités s'offrent à nous: $\mathrm{I}^{\circ}$ l'enceinte sacrée du Temple de Jérusalem; l'autel auprès duquel la foule s'active et sacrifie serait alors l'autel des sacrifices situé devant le Temple et donc à l'intérieur de l'enceinte ; $2^{\circ}$ le Temple lui-même et l'autel serait situé devant lui ; $3^{\circ}$ la maison privée mise à la disposition

\footnotetext{
${ }^{9}$ Plusieurs prépositions pourraient convenir: N2HTC «à l'intérieur », MMdY « là » ou Na2PdC « devant lui ».
} 
du culte chrétien, et l'autel serait alors situé à l'intérieur io. Il est difficile de choisir l'une de ces trois possibilités au détriment des deux autres. Toutefois, même si nous pouvions lire de manière sûre la préposition manquante, le référent exact de la maison et de l'autel ne serait pas assuré pour autant; l'auteur aurait en tête plusieurs référents. En effet, nous estimons qu'il faut tenir compte de deux contextes historiques, à la fois celui dans lequel l'auteur écrit et celui au cours duquel il place sa fiction. Dans le second cas, il n'existe pas de bâtiment cultuel chrétien (et pour cause), mais le Temple de Jérusalem, avec ses deux autels, est le lieu de culte que Jésus et ses disciples fréquentent. Dans le premier cas, le Temple de Jérusalem n'existe plus, détruit en 70 après J.-C. ; son souvenir est néanmoins toujours présent, et, s'il n'existe toujours pas de bâtiment cultuel chrétien proprement dit ${ }^{\mathrm{II}}$, les chrétiens se réunissent dans des lieux privés - i் KaT'oĩkov

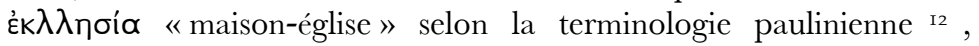
maisons que des particuliers mettent à la disposition de leur communauté - ou des lieux semi-privés - comme des entrepôts dans les Actes apocryphes de Paul ${ }^{\text {I3 }}$. Nous pensons donc que les mentions de l'autel et de la maison doivent être comprises non seulement dans le contexte des premières communautés chrétiennes du II siècle mais aussi dans celui du judaïsme du temps de Jésus : la maison ferait référence à l'une de ces maisons privées mentionnées ci-dessus tout en étant, dans le même temps, assimilée au Temple de Jérusalem ; l'autel qui est en rapport avec cette maison se réferrerait à la fois à l'autel de l'eucharistie situé dans le lieu de culte chrétien, à l'autel des encens, situé à l'intérieur du Temple de Jérusalem, et surtout à l'autel des sacrifices, situé à l'extérieur, avec une confusion volontaire entre les deux derniers. En entretenant un certain flou et une confusion sur les

io Pour le détail, voir VAN DEN Kerchove A., « La maison, l'autel et les sacrifices », op. cit., p. 313-318.

II L'église comme bâtiment dédié au culte n'existe pas avant l'ère constantinienne. Cf. WHITE L. M., « House churches », Oxford Encyclopedia of Archaeology in the Near East, Meyers E. M. (ed.), Oxford, Oxford University Press, I997, vol. 3, p. I I9-I20.

${ }_{12}$ Rom I6.5 ; I Cor. I6.19; Phlm I.2.

I3 Il s'agit plus particulièrement de la dernière partie de cet ouvrage, que le témoignage de Tertullien (dans De Baptismo I7) permet de dater du II ${ }^{\mathrm{e}}$ s. ap. J.-C., partie intitulée Le martyre de Paul. Dans Le martyre de Paul I, on apprend que Paul loua, en dehors de Rome, une grange - öppiov en grec et horreum dans la version latine - pour y enseigner «la parole de vérité ». Lipsius R. A. et Bonnet M., Acta apostorum apocrypha, Hildesheim, G. Olms, I959 (I89I-I903), t. I, p. I04. Voir aussi, « Fiches signalétiques - Les Actes de Paul ", dans Bovon Fr., van EsBroeck M. et alii, Les actes apocryphes des Apôtres. Christianisme et monde païn, Paris, Labor et Fides, I98 I, Publications de la Faculté de théologie de l'université de Genève n ${ }^{\circ}$, p. 295-298. 
référents exacts qu'il a en tête, l'auteur aurait une attitude polémique dès le début de la vision des disciples : il assimile les adversaires qu'il combat, des chrétiens, et leurs activités respectivement à des juifs et aux pratiques rituelles juives telles qu'elles étaient pratiquées au I ${ }^{e r}$ siècle, c'est-à-dire les sacrifices. Certes, ces pratiques n'existent sans doute plus à l'époque de la rédaction de l'Évangile de Judas, le II ${ }^{\mathrm{e}}$ siècle, en raison de la destruction du Temple en 70 ; néanmoins, leur souvenir est conservé vivant par la communauté juive, notamment grâce à la Mishnah (dont la rédaction s'étend de la fin du I ${ }^{e r}$ siècle jusqu'à $2 \mathrm{I} 9$ au plus tard) et à travers d'autres actes rituels juifs, comme la prière des Dix-huit bénédictions, les quatorzième et dix-septième bénédictions portant sur la reconstruction du Temple de Jérusalem et la restauration des cultes ${ }^{\mathrm{I}}$.

\section{Discrédit des mœurs, discrédit des rites}

Venons en maintenant aux pratiques que la foule met en oeuvre. Nous avons dit que ces pratiques explicitent le verbe прОскגртере (et ceci est également valable s'il s'agit non pas de la foule mais des prêtres). Le choix de ce verbe semble résulter d'une intention polémique. L'auteur reprend un verbe qui devient courant dans la littérature chrétienne alors qu'il était plutôt rare auparavant. Cependant, il se singularise par l'usage qu'il en fait ${ }^{15}$. Alors que ce verbe est généralement mis en rapport avec la prière ou l'eucharistie, l'auteur l'associe à l'autel et joue sur le lien métaphorique établi entre d'une part les prières et l'eucharistie et d'autre part le sacrifice, manière de critiquer l'une ou l'autre de ces pratiques, et même les deux, ou plutôt la manière dont les adversaires de l'auteur, des chrétiens, pratiquent ces deux rites. La liste des actions est un moyen d'accentuer cette critique, tout en l'étendant à d'autres pratiques.

La liste, à travers son organisation, vise à critiquer l'ensemble du mode de vie des adversaires de l'auteur, aussi bien leur vie religieuse avec les pratiques rituelles, que leurs mœurs, avec les actions non rituelles, tout en assimilant les premières aux secondes (cf. supra). Critiquer les mœurs des adversaires est une pratique courante, notamment dans la polémique entre les chrétiens et les païens et entre les chrétiens eux-mêmes ${ }^{16}$. Même si, dans l'ÉfJudas, la liste est restreinte comparativement à d'autres listes, on y retrouve les deux grandes catégories de crimes, à savoir les actes illégaux (meurtres,

${ }^{14}$ Massonnet J., «Le sacrifice dans le judaïsme », Cahiers Évangile i i8, 2002, p. 5 et I I-I 2.

${ }^{15}$ Voir le détail de l'argumentation dans VAN DEN Kerchove A., « La maison, l'autel et les sacrifices », op. cit., p. 320-322.

${ }^{16}$ Le Boulluec A., La notion d'hérésie dans la littérature grecque II ${ }^{e}-I I I^{e}$ siècles. Tome I. De Justin à Irénée, Paris, Études augustiniennes, I985, p. I29, I33-I34. 
péchés et actions illégales) et les actes contre nature (dormir avec des hommes). Cette liste, avec seulement trois actes différents, n'est pas close, et la mention finale d'«un grand nombre (OYMHH(1)e) de péchés et d'actions illégales » renvoie à de très nombreux autres actes illégaux mentionnés dans d'autres listes ou textes contemporains plus détaillés ${ }^{17}$. Comme unique exemple de péchés contre nature, l'auteur n'a retenu que la pédérastie : il l'évoque de manière "sobre" (s'il est permis de parler ainsi) - «d'autres dorment avec des hommes »- sans évoquer d'autres actes qui sont souvent mentionnés en même temps que la pédérastie, tels que l'adultère ou l'inceste ${ }^{18}$. L'accusation de pédérastie fait partie de tout un arsenal polémique païen puis chrétien. Les auteurs chrétiens reprennent cette critique qui leur était adressée par les païens pour mieux la retourner contre eux : « eux (les païens), les adultères et les pédérastes, accusant les hommes voués à la continence et au mariage unique $»^{19}$ et citent régulièrement une parole de Paul, Rom. I.26-27 :

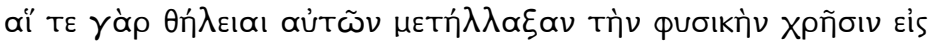

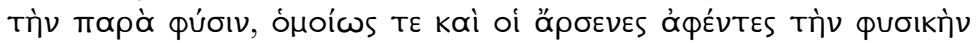

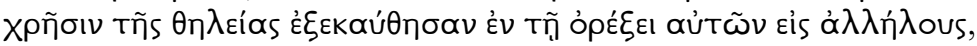

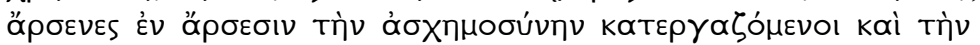

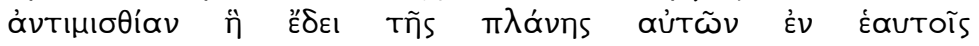

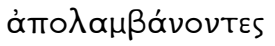

«car leurs femmes remplacèrent la relation naturelle pour une relation contre nature ; de même, les hommes, abandonnant la relation naturelle avec la femme, enflammés dans leur désir les uns pour les autres, les hommes accomplissant l'indécence avec les hommes et recevant en eux-mêmes le salaire qui convient à leur erreur ${ }^{20}$. »

Une telle accusation est aussi utilisée au sein de polémiques chrétiennes, entre les différentes communautés chrétiennes, et le texte de l'ÉvJudas en est un témoignage supplémentaire.

$\mathrm{Au}$ discrédit des mœurs s'ajoute celui des pratiques rituelles. Le choix du verbe PӨYCldCE, "sacrifier», au coeur des pratiques rituelles et en relation avec l'autel, est particulièrement significatif. La mention des sacrifices des enfants et des femmes a une charge polémique très forte. C'est une manière traditionnelle de caractériser

${ }^{17}$ Méliton de SARdes, Sur la Pâque 50-5I ; Théophile D’Antioche, Trois livres à Autolycos III, 3-4 ; ATHANASE D'AleXaNDrIE, De Incarnatione 5.4-5. ${ }^{18}$ Méliton de Sardes, Sur la Pâque 53 ; Athénagore, Supplique au sujet des Chrétiens 34.3.

19 Athénagore, Supplique au sujet des Chrétiens 34.3: kakí̧ovtes oi

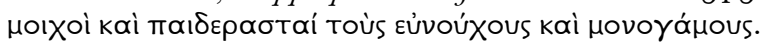

20 Athénagore, Supplique au sujet des Chrétiens 34.2; Athanase

D'AleXANDRIE, De l'incarnation 5.5. 
le barbare et de diffamer l'ennemi et ses adversaires ${ }^{21}$. Si l'idée de diffamation est sûrement bien présente dans l'esprit de l'auteur, faut-il n'y voir que cela? L'auteur ne parlerait-il pas de tels sacrifices en pensant aussi à des pratiques bien précises, ce qui n'était pas toujours le cas dans d'autres utilisations polémiques de cette accusation ${ }^{22}$ ? Ce qui nous incite à envisager une réponse positive à cette dernière question est la propension des chrétiens, les auteurs des livres devenus canoniques et surtout les Pères de l'Église, à avoir recours au langage sacrificiel, notamment pour évoquer l'eucharistie, la continence et le martyre, et ceci surtout de manière métaphorique ${ }^{23}$. L'auteur de l' $E_{V} J u d a s$ reprendrait ce langage métaphorique et le détournerait de sa signification originelle pour mieux le retourner contre ses adversaires, qui devaient eux aussi probablement utiliser un tel champ lexical. Ainsi, en parlant des sacrifices d'enfants et de femmes, l'auteur serait-il en train de critiquer respectivement l'eucharistie et la continence. Dans le même temps, il peut aussi critiquer une seule et même pratique : le martyre ${ }^{24}$. Son procédé revient à envisager littéralement des métaphores chrétiennes afin de mieux accuser ses adversaires des pires maux. Ce procédé est accentué par le fait que la mention des sacrifices humains est encadrée par celle de pratiques bien attestées et réellement mises en oeuvre par les adversaires, le jeûne (si la restitution proposée plus haut est acceptée), les louanges et l'humilité. Ces trois pratiques fonctionnent comme des garants de la véracité des sacrifices tout en étant elles-mêmes «éclaboussées » par l'ignominie que constituent les sacrifices humains.

Avec cette liste, mentionnant des actions en relation avec l'autel, l'auteur dresse une vision bien négative de la foule et des prêtres. De plus, dans sa stratégie, nous pouvons déceler une certaine ironie : les disciples sont en train de critiquer leurs propres pratiques rituelles et

${ }^{2 I}$ VAN HAEPEREN Fr., « Sacrifices humains et mises à mort rituelles à Rome : quelques observations", Folia Electronica Classica 8, 2004 : http://wwwbcs.fltr.ucl.ac.be/FE/o8/sacrifices.htm.

${ }_{22}$ Par exemple, les accusations portées contre Catilina et sa bande par plusieurs auteurs (Plutarque, Cic. I0.4 et Dion Cassius 37, 30.3), accusations qui relèveraient surtout d'une propagande diffamatoire, comme semble déjà le penser Salluste, Catilina 22.I-2.

${ }_{23}$ Hermas, Le pasteur; Tertullien, À son épouse VI I ; Martyre de Polycarpe XIV I. Voir parmi une bibliographie importante : FERGUSON E., "Spiritual Sacrifice in Early Christianity and its Environment », ARNW II, 23.2, I980, p.II63-I I89; Young Fr. M., The Use of Sacrificial Ideas in Greek Christian Writers form the New Testament to John Chrysostom, Cambridge (Massachusetts), The Philadelphia Patristic Foundation, I979, Patristic Monograph Series, $\mathrm{N}^{\circ} 5$, p. 97 et s.

${ }^{24}$ Pour les détails de l'utilisation polémique du verbe « sacrifier » par l'auteur, voir VAN DEN Kerchove A., "La maison, l'autel et les sacrifices », op. cit., p. 325-328. 
leurs propres mœurs quand ils énumèrent ces actions, alors qu'ils se voient eux-mêmes s'activer auprès de l'autel et que Jésus va les identifier aux prêtres.

De la critique du mode de vie à une critique ecclésiologique

Après que les disciples ont exposé leur vision, Jésus en donne une interprétation ; il propose notamment une identification pour chacun des protagonistes et des éléments intervenant dans la vision, en ÉvJudas 39.18-40.2 :

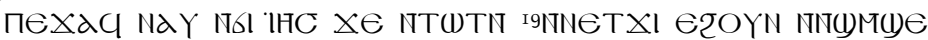
${ }^{20}$ ene

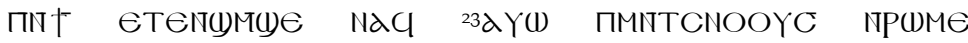

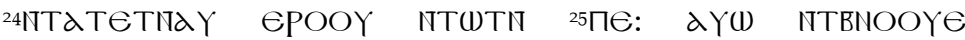

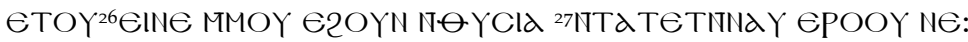

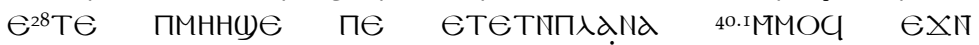

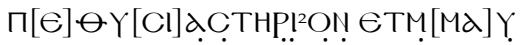

"Jésus leur dit: “vous, vous êtes ceux qui présentent les offrandes sur l'autel que vous avez vu; celui-ci, c'est le dieu que vous servez et les douze hommes que vous avez vus, c'est vous; et le troupeau qui est amené à l'intérieur, c'est les sacrifices que vous avez vus, c'est-à-dire la foule que vous égarez devant cet autel." »

Après avoir assimilé les disciples aux prêtres, Jésus affirme que la foule guidée par ces prêtres est le troupeau mené au sacrifice. Avec cette remarque mise dans la bouche de Jésus, l'auteur revient sur le thème du sacrifice. À quels sacrifices fait-il référence? S'agit-il des sacrifices des femmes et des enfants? Jésus parle d'un troupeau que les disciples auraient vu: ceci ne peut se référer qu'aux seuls enfants et femmes mentionnés par les disciples en relation avec les sacrifices. Toutefois, ceci nous semble être trop restrictif, puisque le troupeau est identifié à la foule avec le même terme copte (MHH(1) ) qui a déjà été utilisé précédemment pour la foule qui s'active auprès de l'autel. On peut se demander si l'auteur ne reprendrait pas ici une appellation parfois utilisée pour désigner les membres de la communauté chrétienne: «troupeau du Christ» ${ }^{25}$; cependant, comme pour le langage sacrificiel, il le fait dans une intention polémique : ce troupeau n'est pas guidé vers le salut, mais vers l'erreur et le sacrifice. Ce dernier ne renverrait pas seulement à celui des enfants et des femmes mais à toutes les pratiques rituelles et les actions accomplies par la

${ }^{25}$ Cette désignation n'est pas très courante dans la littérature chrétienne ; nous la retrouvons chez un auteur contemporain de l'auteur de notre apocryphe, Clément de Rome, dans son Épître aux Corinthiens I 44.2, 54.I et 57.I. Nous la retrouvons un peu plus tard dans deux lettres de Basile de Césarée, les Lettres 90.2 et I97.I. 
foule qui suit les disciples. L'ensemble de la vie religieuse et du mode de vie des adversaires est de cette manière critiqué.

Néanmoins, derrière cela, nous pouvons aussi déceler une critique ecclésiologique. En effet, l'auteur de l'É Evudas polémiquerait moins contre ceux qui mettent en oeuvre ces pratiques que contre ceux qui poussent à mettre en oeuvre de telles pratiques, c'est-à-dire les prêtres. L'auteur parle de ces derniers en ces termes, $E_{V} J u d a s$ 38.24-39.3 : «les hommes qui se tiennent debout devant l'autel, c'est ton $\mathrm{n}$ [om] qu'ils invoquent; et c'est alors qu'ils sont parmi toutes les actions de leur déficience que cet [autel] est complété ». Ces propositions laissent entendre que toutes les actions accomplies par la foule le sont sous l'égide des prêtres, lesquels sont ainsi désignés comme les principaux responsables. Ainsi, d'après ce que dit l'auteur de cet apocryphe, les disciples-prêtres sont-ils loin de ressembler aux prêtres de l'Épître aux Corinthiens de Clément « qui ont rempli leur office envers le troupeau du Christ sans mériter de blâme, avec humilité, calme et dignité, et qui ont longuement reçu le témoignage de tous ${ }^{26}$ ou ceux de la Tradition apostolique $^{27}$. Leur sacerdoce est en effet loin d'être sans reproche. Derrière l'emploi d'un langage sacrificiel pour désigner des pratiques controversées, il faudrait donc comprendre une critique ecclésiologique : l'auteur porte un jugement négatif sur I) la manière dont ces prêtres pratiquent le rite eucharistique, 2) celle dont ils prient et prônent la virginité ainsi que la continence et sur 3) le fait qu'ils guident leurs coreligionnaires vers le martyre, rôle qu'ils peuvent avoir, comme en témoigne la Lettre 76 de Cyprien ${ }^{28}$. Finalement, l'auteur se battrait contre des gens qui se disent prêtres mais qui, selon lui, ne le seraient pas. Pour lui, ce sont de faux prêtres qui guident vers l'erreur,

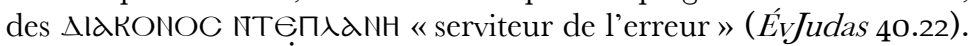
Cette expression rappelle des expressions équivalentes dans un autre texte gnostique, l'Apocalypse de Pierre, NH VII, 3: "père de leur erreur» (73.27) et «messagers de l'erreur» (77.24). L'auteur de cette

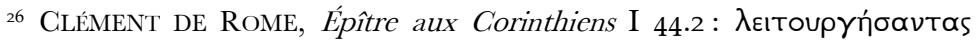

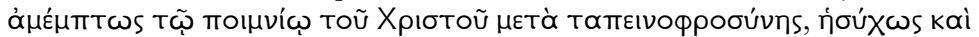

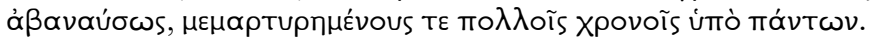

27 Tradition apostolique 3: «accorde, Père, qui connais les cœurs, à ton serviteur que tu as choisi pour l'épiscopat, qu'il fasse paitre ton saint troupeau et qu'il exerce à ton égard le souverain sacerdoce sans reproche, en te servant nuit et jour ».

${ }^{28}$ Cyprien, Lettre 76.4, s'adressant à des évêques, à ses collègues dans le sacerdoce, aux diacres : «et, pour que rien ne manque à l'exemple que nous donnez, au moment même que vous proclamez votre foi, et que vous souffrez pour elle, vous appelez vos frères au divin martyre, et vous avancez les premiers sur la voie du courage : de cette façon le troupeau suit ses pasteurs et imite ce qu'ils font, et il sera lui aussi couronné par le Seigneur pour ses mérites ». 
Apocalypse polémique contre les évêques et les diacres; mais cette critique porterait moins sur ces offices eux-mêmes que sur le fait qu'ils ne tiendraient pas leur autorité de Dieu ${ }^{29}$. Ce rapprochement ne présage pas d'un emprunt ou d'une influence mais d'un terreau commun critique, vis-à-vis de la Grande Église et de sa hiérarchie qui en cours d'élaboration. Dire que les disciples appelés par ailleurs "prêtres », OYHHB, sont aussi des diaconoi est possible à une époque où ce sont deux ordres distincts et non hiérarchiques, c'est-à-dire avant que la Grande Église ne se réalise vraiment avec une hiérarchie tripartite - évêques, prêtres, diacres. De plus, les disciples-prêtres de l'ÉVJudas semblent avoir une fonction collégiale, comme c'est le cas des presbytres jusqu'au début du $\mathrm{III}^{\mathrm{e}}$ siècle ${ }^{30}$.Ceci pourrait nous autoriser à dater la rédaction de l' $E_{V} J u d a s$ au plus tard à la fin du II ${ }^{\mathrm{e}}$ ou au début du III ${ }^{\mathrm{e}}$ siècle, de la même manière que l'Apocalypse de Pierre peut être datée soit du début du $\mathrm{III}^{\mathrm{e}}$ siècle, soit entre les années I5O et $250^{3 \mathrm{I}}$, avant que la fixation de la hiérarchie ecclésiale ne permette plus de confondre, intentionnellement ou non, les deux.

La conclusion logique d'une telle condamnation est donnée par l'auteur à travers la bouche de Jésus, ÉVJudas 4I.I-2 : ₹() $\in \mathrm{P}()^{2} \mathrm{TH}$ $\Pi \Theta[Y$ CldC $\Theta .$.$] «arrêtez de sacrifier ». Jésus appelle donc à la fin des$ sacrifices, c'est-à-dire des pratiques qui sont désignées ainsi, et à la fin de la voie de l'erreur. Cet arrêt pourrait équivaloir à une véritable conversion, à un changement du mode de vie à travers une

${ }^{29}$ Apocalypse de Pierre, NHVII, 79. Voir Koschorke Kl., Die Polemik der Gnostiker gegen das kirchliches Christentum : unter Berücksichtigung der Nag-Hammadi Traktate "Apokalypse des Petrus" NHC VII, 3 und "Testimonium Veritatis" NHC IX, 3, Leyde, E. J. Brill, I978, NHS I2, en particulier p. 8o-84; DesJardins M., "Apocalypse of Peter ", dans Coptic Gnostic Library. A Complete Edition of the Nag Hammadi Codices, RobinsonJ. M. (ed.), Leyde, Brill, 2000, vol. 4, p. 2 I3 n. 44 ; Schweizer E., "The "Matthean" Church», New Testament Studies 20, I974, p. 2 I6 ; idem, «Zur Struktur der hinter dem Mathäusevangelium stehenden Gemeinde », ZNW65, I974, p. I39.

3o Dix G., Jurisdiction in the Early Church. Episcopal and Papal, London, Faith House, 1975, p. 23-46. Lemaire A., Les ministères aux origines de I'Église : naissance de la triple hiérarchie : évêques, presbytres, diacres, Paris, Cerf, I97I, Lectio divina 68, en particulier p. I77-I78; BEYER H. W.,

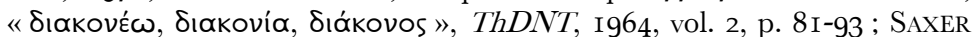
V., "L'organisation des Églises héritées des apôtres (70-I80) », dans Histoire du christianisme. Tome I : Le Nouveau Peuple (des origines à 250), MAYEur J.-M., Pietri CH. et L., Vauchez A. et Venard M. (dir.), Paris, Desclée, 2000, p. 43I-433 et idem, "Le progrès de l'organisation ecclésiastique de la fin du II ${ }^{2}$ siècle au milieu du III ${ }^{\mathrm{e}}$ siècle (I80-250) », dans ce même volume, p. 809 .

${ }^{\text {I }}$ Desjardins M., « Apocalypse of Peter », op. cit., p. 2 I4. 
transformation des pratiques. Toutefois, cet ordre de Jésus ne signifie pas que l'auteur ne revienne plus sur les sacrifices.

Le sacrifice de Judas

En effet, il en parle de nouveau, à la fin du texte, en $E_{V} J u d a s 56$. I I2I :

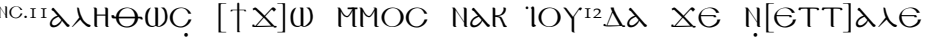

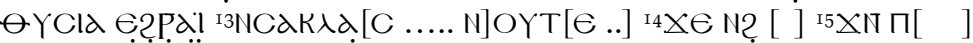

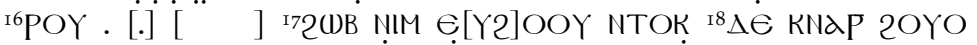

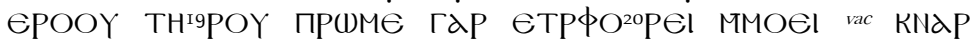
ӨYCldCe ${ }^{2 \mathrm{M} M M O C}$

«En vérité, [je] te [di]s, Judas : «ceux qui offrent des sacrifices à Sakla[s ..... dieu] [ 3 lignes lacunaires ] toute chose mauvaise. Mais, quant à toi, tu feras plus qu'eux tous ; en effet, l'homme qui me porte, tu le sacrifieras. »

Comme nous l'avons déjà dit, dans ce second passage, c'est Jésus qui prend l'initiative de parler des sacrifices, tout en s'adressant à Judas. Dans un premier temps, il évoque «ceux qui offrent des sacrifices à Sakla $[\mathrm{s}]$ » et il fait probablement référence aux sacrifices de la foule mentionnés dans le premier passage. La mention de Saklas renforce la pointe polémique déjà entrevue pour les sacrifices des pages 38-4I : la foule croit mettre en oeuvre des pratiques en l'honneur de Dieu et de Jésus, alors qu'en réalité ils s'adressent à Saklas, un archonte; les disciples ne sont pas les prêtres de Jésus, contrairement à ce qu'ils pensent, mais ceux de Saklas. Dans un second temps, après un passage lacunaire, il annonce à Judas un nouveau sacrifice à venir: celui que Judas devra accomplir sur la personne humaine de Jésus. Jésus situe ce sacrifice sur une échelle de valeur: «tu feras plus qu'eux tous». Quelle connotation faut-il donner à 2OYO «plus »? Terme neutre, son sens précis dépend du contexte. Or, dans le cas de notre apocryphe, le contexte est lacunaire. Néanmoins, il semble que «eux tous » fait référence aux autres disciples-prêtres et à la foule, et, vu la connotation négative attachée aux actions de ces derniers, il en irait de même pour Judas : ainsi, son l'action sera-t-elle pire que celles commises par tous les autres, c'est-àdire les sacrifices de la foule et des disciples-prêtres. La suite explicite la nature de l'action de Judas : le sacrifice de l'homme qui porte Jésus ; à nouveau, il s'agit d'un sacrifice humain. Parler de la mort de Jésus en terme sacrificiel n'est pas nouveau. Paul, en particulier dans sa Première Épître aux Corinthiens 5. I, utilise explicitement ce langage ${ }^{32}$. Si un tel usage devient courant à partir du III $^{e}$ siècle, au $\mathrm{II}^{\mathrm{e}}$ siècle il

${ }^{32}$ QueSnel M., "La sacrifice chez Paul », Cahiers Évangile, I I8, 2002, p. I4i6 ; Berder M., «Le sacrifice dans l'Épître aux Hébreux », Cahiers Évangile, II 8,2002 , p. 3I-38. 
n'est pas sûr qu'il fasse partie du langage chrétien commun 33. Quoi qu'il en soit, l'auteur de l'ÉEJudas adopte le vocabulaire sacrificiel pour la mort de Jésus avec trois idées qui lui sont particulières - ces idées ont pu apparaître ultérieurement comme des distorsions par rapport à la métaphore qui devient courante au cours du III ${ }^{\mathrm{E}}$ siècle. I) Le sacrifice commis par Judas se réfere moins à la mort de Jésus luimême qu'à la trahison de Jésus par Judas. Certes, celle-ci va conduire à la crucifixion de Jésus, et ainsi Judas, comme les prêtres qui officient sont responsables des sacrifices de la foule, est-il responsable de la mort de Jésus.

2) Judas sacrifie moins Jésus lui-même que «l'homme qui me [Jésus]

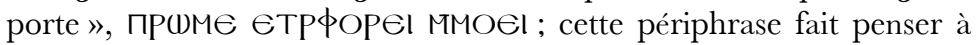
un terme ultérieur, хрібтофópos, qui désigne un chrétien, un homme inspiré par le Christ ou, encore, qui est une métaphore pour le corps du martyr ${ }^{34}$, ce qui peut être intéressant si l'on accepte l'hypothèse selon laquelle un référent des sacrifices humains de la page 38 pourrait être le martyre. L'auteur de notre texte aurait donc une interprétation docète de la mort de Jésus.

3) Il établit une équivalence entre les sacrifices de la foule sous l'égide des prêtres et le sacrifice de Judas : tous sont des sacrifices humains, ils font référence à des pratiques ou des événements réels et sont mauvais par nature. Néanmoins, une différence importante est introduite subtilement (du moins, le pensons-nous) : l'opposition entre le sacrifice unique et les sacrifices multiples permet en définitive d'opposer Judas aux autres disciples-prêtres, et cette opposition serait accentuée par l'emploi de 2OYO «plus ». Avec cet emploi, l'auteur opère une sorte de renversement de valeur du sacrifice accompli par Judas : en faisant plus que tous les autres, en accomplissant le plus grand sacrifice qui puisse être, il commet le dernier sacrifice, celui qui ne pourra pas être dépassé et qui mettra donc fin à tous les sacrifices. L'auteur reprend l'interprétation courante de la mort de Jésus comme le sacrifice ultime qui met fin à tous les sacrifices, interprétation qui donne une valeur positive à un événement déroutant, et il transpose cette ambivalence à la figure de Judas. Ainsi, estimons-nous que dans ce texte, la figure de Judas n'est ni bonne ni mauvaise; elle serait simplement utile grâce à son ambivalence, car une action mauvaise ouvre une nouvelle ère avec de nouvelles pratiques.

33 Chauvet L.M., «Le "sacrifice” en christianisme : une notion ambiguë », dans Neusch M. (ed.), Le sacrifice dans les religions, Paris, Beauchesne, I994, Sciences théologiques et religieuses 3, p. I47 et s.; Young Fr.M., op. cit., p. I3I-I35; FERGUSON E., op. cit., p. I I63 et s.

34 Danker Fr. W., A Greek-English Lexicon on the New Testament and other Early Christian Literatur, ze edition based on the sixth edition of W. Bauer's Griecisch-Deutsches Wörterbuch, Chicago - London, The University of Chicago Press, 2000, p. IogI. 


\section{Conclusion}

Nous espérons avoir montré, dans les pages précédentes, l'importance du thème sacrificiel dans ce nouveau texte gnostique. L'emploi du langage sacrificiel ne serait pas une simple façon de parler, mais relèverait d'une certaine stratégie polémique de l'auteur contre d'autres communautés chrétiennes dont il critiquerait les pratiques et l'organisation ecclésiale. Pour cela, il reprend le langage métaphorique de son époque en feignant de le prendre à la lettre et rappelle, avec une nouvelle interprétation, des figures et des événements du début du $\mathrm{I}^{\mathrm{er}}$ siècle. Tout ceci contribuerait finalement à mettre en valeur la figure de Judas. Toutefois, cette valorisation doit être nuancée: il s'agirait plutôt d'accentuer l'ambivalence de ce personnage, à l'image de l'ambivalence de la mort de Jésus vécue à la fois comme un événement tragique et comme le début d'une ère nouvelle. Toutes ces remarques peuvent-elles contribuer à mieux comprendre l'intention d'un tel ouvrage ? Il est encore trop tôt pour le dire, d'autant que les hypothèses que nous avançons ne sont pas définitives et sont loin d'avoir épuisé le sujet. 